\title{
Transient Magnetic Birefringence for determining magnetic nanoparticle diameters in dense, highly light scattering media
}

Running head: Application of Transient Magnetic Birefringence to Dense, Highly Light Scattering Media

\section{Mariana Köber, ${ }^{1}$ Maria Moros, ${ }^{2}$ Valeria Grazú, ${ }^{2}$ Jesus M. de la Fuente, ${ }^{2}$ Mónica Luna, ${ }^{1}$ and Fernando Briones ${ }^{1}$}

\author{
${ }^{1}$ IMM-Instituto de Microelectrónica de Madrid (CNM-CSIC), Isaac Newton 8, PTM, \\ E-28760 Tres Cantos, Madrid, Spain \\ ${ }^{2}$ Instituto de Nanociencia de Aragón, University of Zaragoza, Campus Río Ebro, Edif. \\ I+D c/ Mariano Esquillor, 50018 Zaragoza, Spain
}

E-mail: mariana.kober@csic.es

\begin{abstract}
The increasing use of biofunctionalized magnetic nanoparticles in biomedical applications calls for further development of characterization tools that allow for determining the interactions of the nanoparticles with the biological medium in situ. In cell-incubating conditions, for example, nanoparticles may aggregate and serum proteins adsorb on the particles, altering the nanoparticles' performance and their interaction with cell membranes. In this work we show that the aggregation of spherical magnetite nanoparticles can be detected with high sensitivity in dense, highly light scattering media by making use of magnetically induced birefringence. Moreover, the hydrodynamic particle diameter distribution of anisometric nanoparticle
\end{abstract}


aggregates can be determined directly in these media by monitoring the relaxation time of the magnetically induced birefringence. As a proof of concept, we performed measurements on nanoparticles included in an agarose gel, which scatters light in a similar way as a more complex biological medium but where particle-matrix interactions are weak. Magnetite nanoparticles were separated by agarose gel electrophoresis and the hydrodynamic diameter distribution was determined in situ. For the different particle functionalizations and agarose concentrations tested, we could show that gel electrophoresis did not yield a complete separation of monomers and small aggregates, and that the electrophoretic mobility of the aggregates decreased linearly with the hydrodynamic diameter. Furthermore, the rotational particle diffusion was not clearly affected by nanoparticle-gel interactions. The possibility to detect nanoparticle aggregates and their hydrodynamic diameters in complex scattering media like cell tissue makes Transient Magnetic Birefringence an interesting technique for biological applications.

PACS: 87.85.Ox, 87.85.Rs, 75.50.Tt, 61.46.Df, 42.25.Lc 


\section{Introduction}

Biofunctionalized magnetic nanoparticles (NPs) are of growing interest for biomedical applications such as magnetic resonance imaging contrast enhancement, immunoassay, hyperthermia, and drug delivery $[1,2]$. For these applications, the particles have to be manipulated in biological environmental conditions, those being buffer, serum or cell tissue. Recent studies confirm that proteins and other biomolecules adsorb to the nanoparticles when those are exposed to biological fluid [3]. Moreover, some aggregation may occur despite adequate particle functionalization and directly affect nanoparticle performance, for example in cell-incubating conditions where nanoparticle aggregation and protein adsorption alter the nanoparticles' interaction with the cell membranes [4]. To control nanoparticle performance in situ, techniques are needed which allow for monitoring the nanoparticles in dense, highly light scattering media.

The hydrodynamic particle diameter is an indicator of particle aggregation and the adsorption of biomolecules, as well as of the conformation of the surfactant molecules and the hydration layer, since it comprises the inorganic core, the surfactant molecules and the solvent layer attached to the particle when it moves. While the geometric size of the inorganic core has been extensively characterized, little is known about the hydrodynamic size of water soluble nanoparticles, even though it is an important parameter for understanding, controlling and optimizing the nanoparticles' performance in biological assays as well as the particles' migration in live cells and tissues. Studies have shown that hydrodynamic radii are strongly affected by nature and lateral extension of the capping ligands and, furthermore, systematically larger than the geometric radii for the inorganic core plus cap, since the coating layer strongly interacts with the surrounding solvent [5]. Since both the conformation of the surfactant molecules [6] and the hydration layer [7] affect the particles' chemical functionality, monitoring the hydrodynamic particle diameter is of major interest in a variety of applications.

In this article we analyze the benefits and drawbacks of using Transient Magnetic Birefringence 
(TMB) to determine the hydrodynamic diameters of magnetite nanoparticles in dense, highly light scattering media. So far, Transient Magnetic Birefringence has only been applied to nanoparticle suspensions [8-10], but never to dense, highly light scattering media. In this work, the method is studied for magnetite nanoparticles included in an agarose gel - a model system in which the viscosity inside the gel cavities is known and the particle interaction with the matrix is weak. As a proof of concept for this technique, nanoparticles of different functionalizations were separated by agarose gel electrophoresis and their hydrodynamic diameters were determined in situ as a function of electrophoretic mobility. In these experiments, we expect that a moderate light depolarization due to multiple scattering has no major effect on the determination of the hydrodynamic radius by means of TMB, since the basis of this method is the analysis of the time dependence of the birefringence decay after the magnetic field is switched off, whereby this birefringence emergence and decay only arises from magnetic nanoparticle alignment and relaxation upon the application of magnetic field pulses, while light depolarization due to background scattering is constant at all times.

We present a systematic study, comparing the hydrodynamic diameter values obtained in situ by TMB with those obtained ex situ by Dynamic Light Scattering (DLS), and correlate both to observations made in microscopy studies (Transmission Electron Microscopy (TEM) and Atomic Force Microscopy (AFM)). In this way we demonstrate that the main contribution to the birefringence signal comes from dimers and higher order aggregates of spherical $\mathrm{Fe}_{3} \mathrm{O}_{4}$ nanoparticles, while monomers do not yield an appreciable birefringence signal. Therefore, magnetically induced birefringence can be used in sensitive sensor applications, where molecular recognition is detected through a significant rise in birefringence through specific and controlled aggregation of adequately functionalized magnetite particles. The presented results pave the way to use magnetically induced birefringence for studying possible interactions of the nanoparticles with their biological surroundings in dense, highly light scattering media like living cells and tissue. 


\section{Transient Magnetic Birefringence}

Transient Magnetic Birefringence (TMB), or Magnetically Induced Birefringence Relaxation (MBR), is based on applying a pulsed magnetic field to a suspension containing magnetic nanoparticles and detecting the induced birefringence by polarimetric measurements $[11,12]$. Nanoparticles and nanoparticle clusters with shape anisotropy will rotate in the solution to align with their long axis in direction of the magnetic field. The necessary coupling of the field induced magnetic moment to the optical anisotropy axis of the nanoparticle depends on magnetic anisotropy, particle size, and temperature. Aggregates yield optical anisotropy due to the oscillating dipole interaction anisotropy which decreases with the cube of the distance between the particles in the aggregate. Also the magnetic dipolar interaction, responsible for the alignment of particle chains with the magnetic field, decreases with the cube of the distance between the particles in the chain. It is worth noticing that during the very short duration of the applied magnetic field pulses (of less than one millisecond) a magnetic field induced aggregation of superparamagnetic nanoparticles is not expected.

Once birefringence is induced, the hydrodynamic diameter of the particles that contribute to the birefringence signal can be determined: Upon removal of the magnetic field, the induced optical anisotropy disappears gradually due to the Brownian rotational motion which the particles undergo in the liquid. This decay is exponential with a characteristic relaxation time dependent on the hydrodynamic particle diameter as well as the viscosity and temperature of the solution. The magnetically induced birefringence (difference in the sample's refractive indices parallel and perpendicular to the magnetic field) decays as [13]

$$
\Delta n(t)=\Delta n_{0} \exp \left(-6 D_{\mathrm{r}} t\right)
$$

where $\Delta n_{0}$ is the birefringence at the time the magnetic field is switched off. For a spherical particle the rotational diffusion constant $D_{\mathrm{r}}$ is given by 


$$
D_{\mathrm{r}}=\frac{k T}{\pi \eta d_{\mathrm{hyd}}^{3}},
$$

where $d_{\text {hyd }}$ is the hydrodynamic particle diameter, $\eta$ the solvent viscosity, $k=1.38 * 10^{-23} \mathrm{~m}^{2} \mathrm{kgs}^{-2} \mathrm{~K}^{-1}$ Boltzmann's constant and $T$ the temperature of the solution.

a

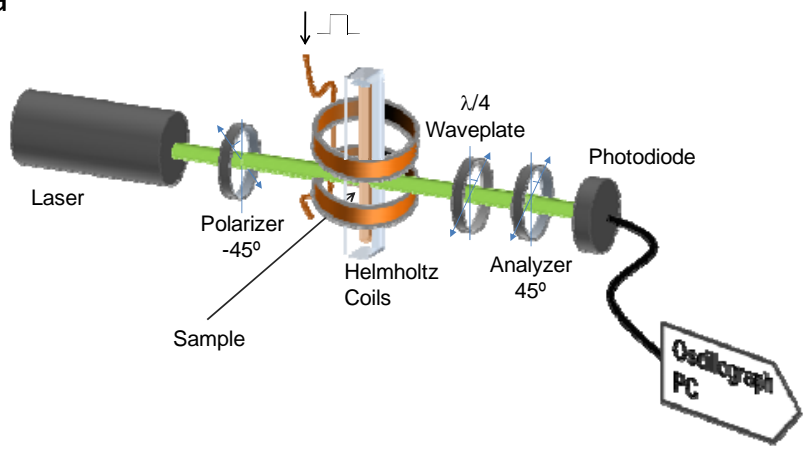

b

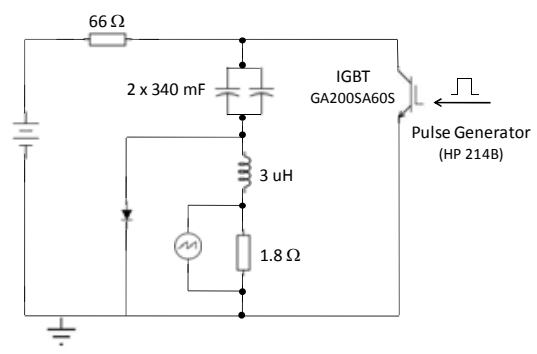

Figure 1. Setup for measuring Transient Magnetic Birefringence. (a) Sketch of the measurement setup; (b) Diagram of the circuit used for powering the Helmholtz coil.

To detect the transient magnetic birefringence the experimental setup sketched in figure 1a was used. On an optical bank a He-Ne laser beam of wavelength $\lambda=543 \mathrm{~nm}$ and low power $(2 \mathrm{~mW})$ was aligned to pass through a polarizer, sample, quarter wave plate and analyzer and to be detected in a photodiode with low noise current to voltage converter. In an oscillograph the signal was averaged over 100 cycles to improve the signal to noise ratio. Polarizer - rotated $45^{\circ}$ respective to the magnetic field direction - and analyzer are crossed, and the direction of the quarter wave plate's fast axis is parallel to the analyzer's polarization axis, as indicated in figure 1a. The sample, fixed in between two non-birefringent glass slides, was submitted to magnetic field pulses produced by a Helmholtz coil. The regime in which birefringence saturates is technically inaccessible since magnetic field pulses have to be not only quite high in order to align even the smallest nanoparticles but also very sharp so that the fall time of the 
magnetic field is much lower than the relaxation time of the particles. In order to create high magnetic fields with low rise and fall times a circuit was designed which employs a transistor IGBT and capacitors, as shown in the circuit diagram in figure 1b. Magnetic field pulses of $55 \mathrm{kA} / \mathrm{m}$ field strength, $5 \mu$ s rise and fall time and $400 \mu$ s pulse width (large enough to assure the alignment of larger particles) were applied, the pulse period being $100 \mathrm{~ms}$.

In this experimental configuration the light intensity detected in the photodiode, $I$, is linear in the birefringence [14], and therefore

$$
I(t)=I_{0} \exp \left(-6 D_{\mathrm{r}} t\right)
$$

where $I_{0}$ denotes the light intensity at the time the magnetic field is switched off. Once the rotational diffusion constant is obtained the hydrodynamic diameter of the particles can be determined with equation (1).

When the particle size distribution cannot be neglected, the birefringence relaxation does not follow a simple exponential decay, and the detected intensity is

$$
I(t)=\int I_{0}\left(d_{\text {hyd }}\right) \exp \left(-6 D_{\mathrm{r}}\left(d_{\text {hyd }}\right) t\right) P\left(d_{\text {hyd }}\right) \mathrm{d} d_{\text {hyd }},
$$

$P\left(d_{\text {hyd }}\right)$ being the probability density function of the particle size distribution. The dependence of $I_{0}$ on $d_{\text {hyd }}$ is weak compared to the dependence of $P$ on $d_{\text {hyd }}$, so $I_{0}$ can be assumed to be constant in a good approximation. Since nanoparticles are generally log-normally distributed [15] we used the log-normal distribution to approximate the particle size distribution. In order to account for the asymmetry of the log-normal distribution we chose the geometric mean and standard deviation (which is multiplicative) as characterization parameters [16]. Then the confidence interval is asymmetric around the mean.

The experimental procedure is straightforward: The sample is inserted in the setup (figure 1a), the magnetic field pulses are turned on and the transmitted light intensity is acquired in a time 
interval of $10 \mathrm{~s}$. A regression of equation (2) to the transmitted light intensity yields the hydrodynamic diameter distribution of the particles.

\section{Materials and methods}

\subsection{Synthesis of $8 \mathrm{~nm}$ iron oxide nanoparticles}

Monodisperse $\mathrm{Fe}_{3} \mathrm{O}_{4}$ nanoparticles of $8 \mathrm{~nm}$ in diameter were synthesized following the seedmediated growth method described by Sun [17]. First, $6 \mathrm{~nm} \mathrm{Fe}_{3} \mathrm{O}_{4}$ nanoparticle seeds were synthesized by mixing and stirring under a flow of argon, $F e(a c a c)_{3} \quad(0.71 \mathrm{~g}), 1,2$ hexadecanediol $(2.58 \mathrm{~g})$, oleic acid $(2 \mathrm{~mL})$ and oleylamine $(2 \mathrm{~mL})$, solubilized in phenyl ether (20 mL). The mixture was heated to $200^{\circ} \mathrm{C}$ for 2 hours and afterwards heated to reflux $\left(265^{\circ} \mathrm{C}\right)$ under argon atmosphere for 1 hour. The mixture was allowed to cool to room temperature and the nanoparticles were washed by adding ethanol and collecting them with a magnet and redispersed in hexane three times. NPs were redispersed in $10 \mathrm{~mL}$ hexane containing $50 \mu \mathrm{l}$ of oleic acid and $50 \mu \mathrm{l}$ of oleylamine. In order to obtain $8 \mathrm{~nm}$ nanoparticles, $80 \mathrm{mg}$ of the $5-6 \mathrm{~nm}$ $\mathrm{Fe}_{3} \mathrm{O}_{4}$ seeds in hexane were added to a mixture containing $\mathrm{Fe}(\mathrm{acac})_{3}(0.71 \mathrm{~g})$, 1,2hexadecanediol $(2.58 \mathrm{~g})$, oleic acid $(0.5 \mathrm{~mL})$, oleylamine $(0.5 \mathrm{~mL})$ and $20 \mathrm{~mL}$ of phenyl ether. The mixture was heated to $100{ }^{\circ} \mathrm{C}$ for $30 \mathrm{~min}$ to remove the hexane and then to $200{ }^{\circ} \mathrm{C}$ for $1 \mathrm{~h}$. Under argon atmosphere the mixture was further heated to reflux $\left(265^{\circ} \mathrm{C}\right)$ for another hour. The mixture was allowed to cool at room temperature by removing the heat source and as described for the $6 \mathrm{~nm}$ nanoparticles, they were precipitated with ethanol and resuspended in hexane. NPs of $8 \mathrm{~nm}$ were precipitated with ethanol and weighed once completely dry. Unless otherwise stated, all the reagents were purchased from Sigma Aldrich. 


\subsection{Water solubilisation of hydrophobic magnetic nanoparticles}

In order to achieve water solubility the synthesized nanoparticles were coated with an amphiphilic polymer shell, namely a poly(maleic anhydride-alt-1-octadecene) (PMAO) shell [18]. To this purpose, $250 \mathrm{mg}$ of PMAO was added to a flask containing $200 \mathrm{~mL}$ of chloroform. After the polymer was dissolved under magnetic stirring, $20 \mathrm{mg}$ of the nanoparticles were added and the mixture was gently stirred for one hour at $25^{\circ} \mathrm{C}$. Afterwards, the solvent was removed under vacuum and few millilitres of chloroform were added. Nanoparticles were then resuspended in $20 \mathrm{~mL}$ of $\mathrm{NaOH} 0.05 \mathrm{M}$ and the sample was shaken at $60^{\circ} \mathrm{C}$ in order to speed up the complete evaporation of chloroform. At this point the solution became clear as NPs were completely transferred into water. NPs were then filtered using syringe filters of $0.22 \mu \mathrm{m}$ to remove aggregates. The NP solution was centrifuged three times at 25,000 rpm for 2 hours to remove the excess unbound polymer molecules. The nanoparticles at the bottom were recovered and redispersed in water. Thus, finally, the nanoparticles present a magnetite core of $8 \mathrm{~nm}$ in diameter and a shell of PMAO of about $3 \mathrm{~nm}$.

\subsection{NPs surface functionalization with galactose and PEG}

A fraction of these particles was modified with galactose (Gal) and another fraction with polyethylene glycol (PEG). To this end, $1 \mathrm{mg}$ of PMAO modified NPs were incubated with $5 \mathrm{mg}$ of N-(3-dimethylaminopropyl)-N'-ethylcarbodiimide hydrochloride (EDC) and $25 \mu$ moles of 4-aminophenyl $\beta$-D-galactopyranoside or $\alpha$-methoxy- $\omega$-amino poly(ethylene glycol) (Rapp Polymere, PEG-MW 750 Dalton) in $250 \mu$ of buffer containing $50 \mathrm{mM}$ boric acid and $50 \mathrm{mM}$ sodium borate (SSB) $\mathrm{pH} 9$. After 2 hours of reaction at room temperature, the NPs were purified of the ligand excess by washing the sample with phosphate buffered saline (PBS) pH 7.4 in a centrifugal filter with a 50,000 Da molecular weight cut off membrane (Millipore). The functionalization of the particles is shown schematically in figure 2a. 


\subsection{Phase Analysis Light Scattering (PALS) measurements}

Zeta potential measurements were performed on the nanoparticles suspended in deionized water at $25^{\circ} \mathrm{C}$ and $\mathrm{pH} 7.4$ on a Zeta PALS instrument (Brookhaven, USA). The given zeta potential values are averages over three measurements (whereat each measurement already forms the average over 10 measurements).

\subsection{TEM conditions}

Size and shape of the nanoparticles' inorganic $\mathrm{Fe}_{3} \mathrm{O}_{4}$ core were characterized by Transmission Electron Microscopy (TEM). A single drop $(10 \mu \mathrm{L})$ of aqueous solution $(0.1 \mathrm{mg} / \mathrm{mL})$ of the nanoparticles was deposited on a copper grid coated with a thin carbon film and let air-dry for several hours at room temperature. TEM analysis was carried out in a JEM-1200EX electron microscope working at $80 \mathrm{kV}$. The particle size distribution was evaluated from several micrographs using an automatic image analyzer. In order to obtain stable size distribution statistics about 100 particles were taken into consideration for this procedure.

\subsection{AFM measurements}

We performed Atomic Force Microscopy (AFM) measurements to prove the presence of aggregates. A drop $(2 \mu \mathrm{L})$ of aqueous NP solution $(0.1 \mathrm{mg} / \mathrm{mL})$ was deposited on a cleaved mica substrate and let air-dry. Measurements were performed with a commercial AFM (Nanotec Electronica Cervantes FullMode AFM System, http://www.nanotec.es/) operated in the dynamic amplitude modulation mode at room temperature and ambient humidity. A second feedback loop was turned on which maintains the phase constant to $90^{\circ}$ ("phase-locked loop") by varying 
the excitation frequency. A monocrystalline silicon cantilever with a force constant of $2.8 \mathrm{~N} / \mathrm{m}$ and a resonance frequency of $75 \mathrm{kHz}$ was used (Nanosensors, Germany).

\subsection{Gel electrophoresis}

We studied the application of TMB to magnetite nanoparticles included in an agarose gel, for which we separated particles by their electrophoretic mobility in the gel. $0.5 \%$ and $2 \% \mathrm{w} / \mathrm{v}$ agarose gels were prepared by mixing $0.5 \mathrm{~g}$ and $2 \mathrm{~g}$, respectively, of agarose (A5093, Sigma) with $100 \mathrm{~mL}$ of $0.5 \mathrm{x}$ Tris-Borate-EDTA buffer (TBE) $\mathrm{pH} 8.7$, obtained from diluting $10 \mathrm{x}$ TBE buffer which was prepared by mixing $108 \mathrm{~g}$ Tris base (93350, Fluka), $55 \mathrm{~g}$ boric acid (B7901, Sigma) and $9.3 \mathrm{~g}$ EDTA (E6635, Sigma) with $\mathrm{H}_{2} \mathrm{O}$ (MiliQ, $18 \mathrm{M} \Omega$ ) until $1 \mathrm{~L}$ was filled. The mixture was heated and let gel in a mold, leaving wells for depositing the suspended NPs. In order to allow for heat dissipation the gel was submerged in the buffer solution with which the horizontal electrophoresis system (Mini-Sub Cell GT, Bio-Rad (electrode spacing of $15 \mathrm{~cm}$ )) was filled. Glycerol (1/5 in volume) was added to the aqueous nanoparticle solution of $0.16 \% \mathrm{w} / \mathrm{v}\left(2140 \mathrm{uM} \mathrm{Fe}_{3} \mathrm{O}_{4}\right)$ and the mixture was loaded in the wells. An electric field of $5 \mathrm{~V} / \mathrm{cm}$ was applied for $105 \mathrm{~min}(0.5 \%$ agarose gel) and $110 \mathrm{~min}$ (2\% agarose gel).

\subsection{Dynamic Light Scattering experiments}

Ex situ Dynamic Light Scattering (DLS) measurements were performed to cross-check the hydrodynamic diameter values determined with TMB. In order to perform DLS measurements on the electrophoretically separated particles the gel lane was cut into three pieces and submerged in TBE $0.5 \mathrm{x}$ buffer solution. After about one week the majority of the particles had diffused out of the gel into the buffer solution. Measurements were performed with a Zetasizer Nano ZS (Malvern Instruments Ltd, United Kingdom) on a series of dilutions of the diffused 
particles in deionized water $\left(T=25^{\circ} \mathrm{C}\right)$. The hydrodynamic particle diameter distribution is given in terms of intensity (figure 4) and number (figure S5 of the Supplementary Data).

\section{Results and Discussion}

Experiments were performed with spherical $\mathrm{Fe}_{3} \mathrm{O}_{4}$ (magnetite) nanoparticles with different functionalizations [19]. Magnetite nanoparticles are widely employed in biomedical applications due to the ease of fabrication and their low toxicity. Monodisperse spherical $\mathrm{Fe}_{3} \mathrm{O}_{4}$ nanoparticles of $8 \mathrm{~nm}$ mean diameter were synthesized following the seed-mediated growth method (figures 3a and S1 of the Supplementary Data) [17]. In order to achieve water solubility the synthesized nanoparticles were coated with an amphiphilic polymer shell, namely a poly(maleic anhydridealt-1-octadecene) (PMAO) shell [18]. PMAO functionalized $\mathrm{Fe}_{3} \mathrm{O}_{4}$ nanoparticles are stable in aqueous solutions - currently available data demonstrate stability for over two years [20]. A fraction of these particles was modified subsequently with galactose (Gal) and another fraction with polyethylene glycol (PEG), as shown schematically in figure 2a.

We studied the application of TMB to magnetite nanoparticles included in an agarose gel, for which we separated particles by their electrophoretic mobility in the gel. Gel electrophoresis, commonly employed to separate biomolecules (DNA, RNA, proteins...), has lately also been applied to the separation of nanoparticles and nanoparticle-biomolecule conjugates according to differences in their size and/or zeta potential (for a recent review see Reference [21]). The electrophoretic particle mobility (= particle velocity / electric field strength) depends on the relation of the particle size to the gel pore size and on the particles' net charges. When the nanoparticle size is very small compared to the gel pore size, the particles' net charges are determinant for the electrophoretic mobility. In this case the electrophoretic mobility may increase with particle size [22]. On the contrary, when the nanoparticles are big enough to "sense" the gel pore walls, steric restrictions and an increased hydrodynamic drag caused by the 
pore walls lead to a reduced electrophoretic mobility for bigger particles [23]. Gel electrophoresis has proven useful to separate nanoparticle assemblies according to their size [23], shape [22] or even to the number of functional groups attached to them [6, 24], and the separation quality has been shown to be strongly influenced by the functionalization layer $[23,24]$. Here, a separation of the nanoparticles through their electrophoretic mobility was carried out in agarose gels of $0.5 \%$ and $2 \% \mathrm{w} / \mathrm{v}$ (applying $5 \mathrm{~V} / \mathrm{cm}$ for $105 \mathrm{~min}$ and $110 \mathrm{~min}$, respectively) in $0.5 \mathrm{x}$ Tris-Borate-EDTA buffer (TBE) $\mathrm{pH}$ 8.7. Photographs (figures $2 \mathrm{~b}$ and $2 \mathrm{c}$, true color brightness-enhanced) show that the particles move in a single band for both agarose concentrations.

After electrophoretic separation we used TMB to determine the hydrodynamic particle diameters as a function of electrophoretic mobility in situ (while the particles remained included in the gel). The procedure is detailed and illustrated in the Supplementary Data (figure S2). Results for particles of the three different functionalizations (PMAO, PMAO + Gal and PMAO + PEG) and two different agarose concentrations $(0.5 \% \mathrm{w} / \mathrm{v}$ and $2 \% \mathrm{w} / \mathrm{v})$ are summarized in figure 2. The regions in which the birefringence signal was significant $(>5 \mathrm{mV})$ is marked by a dotted box in the photographs. 


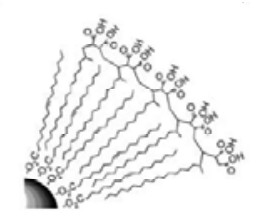

- PMAO

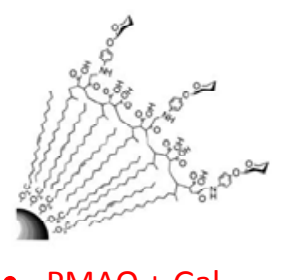

- $\mathrm{PMAO}+\mathrm{Gal}$

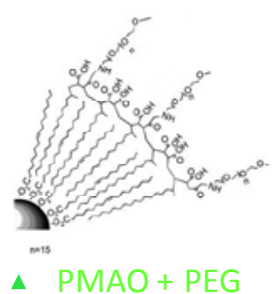

$\triangle \quad P M A O+P E G$

b

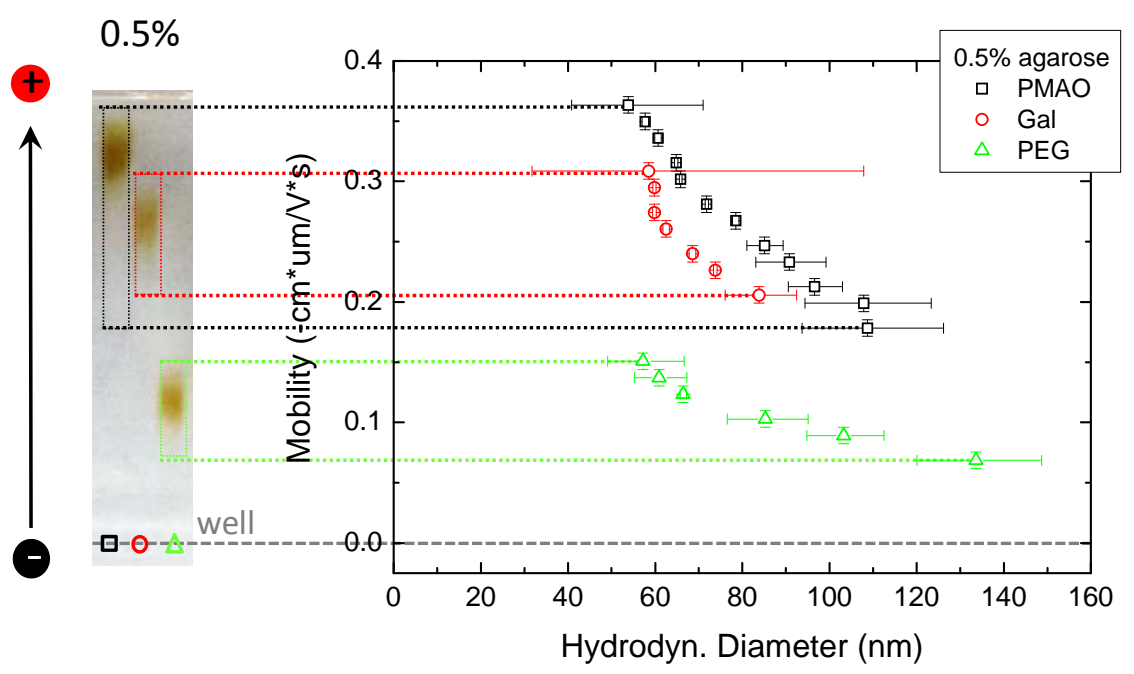

C

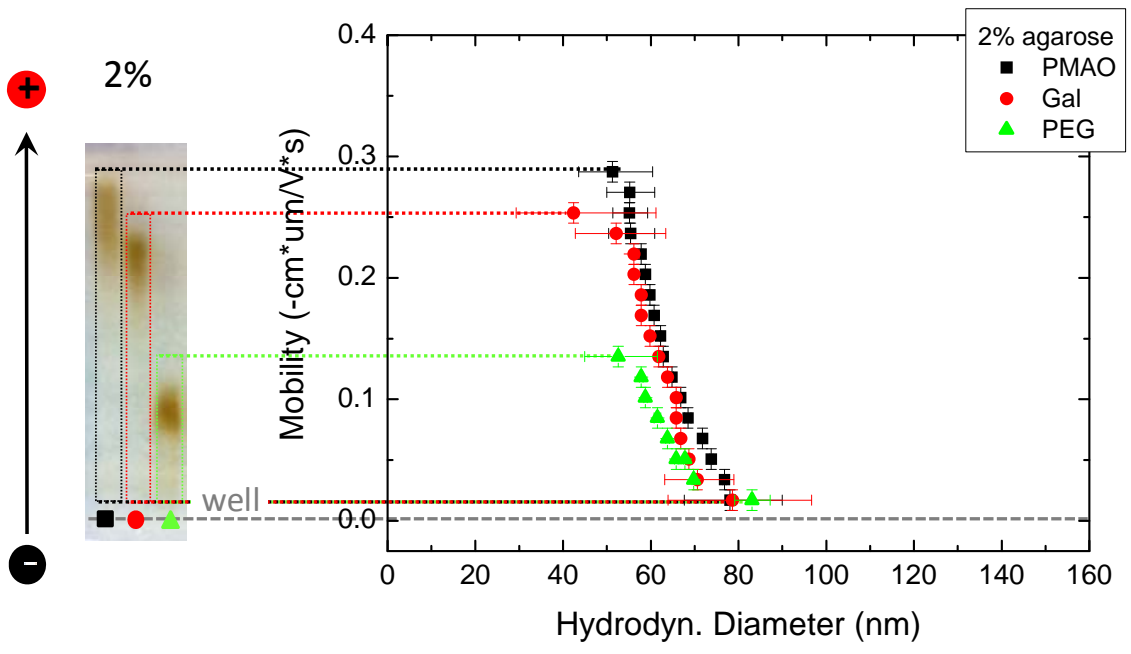

Figure 2. Electrophoretic nanoparticle mobility as a function of the mean hydrodynamic particle diameter determined in situ by Transient Magnetic Birefringence (TMB). (a) Functionalization of the $\mathrm{Fe}_{3} \mathrm{O}_{4}$ nanoparticles (PMAO with optional galactose or PEG modification) (b) and (c) left: Brightness enhanced true color photograph of a $0.5 \%$ and $2 \%$ agarose gel, respectively, after the electric field had been applied. The three lanes contain particles functionalized with PMAO (left lane), PMAO + Gal (middle lane) and PMAO + PEG 
(right lane), as indicated by the symbols; right: Electrophoretic mobility dependence on the hydrodynamic particle diameter, as measured in situ by TMB. Plotted is the geometric mean diameter with the confidence interval of one standard deviation.

The electrophoretic mobility depends approximately linearly on the hydrodynamic diameter, independently of the particle functionalization and agarose concentration. Among the particles of the same functionalization a separation by size occurs, due to a reduced electrophoretic mobility for increasing hydrodynamic diameters. The electrophoretic mobility depends strongly on the agarose concentration, in consequence of the pore diameter. From the finite rotational mobility observed with TMB we can deduce that particles are not trapped in the gel, but rather retained through an increased hydrodynamic drag caused by the pore walls and/or steric restrictions. Only particles with a hydrodynamic diameter $>80 \mathrm{~nm}$ in the $2 \%$ gel are trapped in the first gel section close to the well, in contrast to the $0.5 \%$ gel where aggregates of $140 \mathrm{~nm}$ pass. Unlike for the electrophoretic mobility, we did not find clear evidence that the rotational mobility depends on the gel pore size: the lowest detected hydrodynamic diameter is very similar for both agarose concentrations, and results of in situ and ex situ measurements do not show an unambiguous trend (figure S3 in the Supplementary Data). The particle size distribution width, in turn, depends again clearly on the gel pore size, as the confidence intervals of one standard deviation, represented through $\mathrm{x}$-bars in the graphs, show. While for a $0.5 \%$ gel the size distribution width decreases slowly with increasing traversed distance, for a $2 \%$ gel it is negligibly small even for particles that have hardly traversed any distance in the gel. At the forefront of the band (high electrophoretic mobility) some size dispersion remains for both agarose concentrations. Smaller gel pores would be necessary to achieve a full size separation.

The differences in electrophoretic mobility resulting from modifying the particle functionalization can be attributed mainly to the differences in the particles' net charges since the hydrodynamic particle diameters are very similar for the different types of functionalization. 
This tendency is reflected in the zeta potentials determined in water, which resulted to be $-53 \mathrm{mV}$ for the PMAO functionalized particles, $-32 \mathrm{mV}$ for the particles modified with galactose and $-1.8 \mathrm{mV}$ for those modified with PEG. The deprotonation of the PMAO carboxilic groups in the basic environment of the TBE buffer $(\mathrm{pH} \approx 9)$ is responsible for the PMAO coated particles to be negatively charged. By adding galactose or PEG molecules to the coating layer, charge and, therewith, electrophoretic mobility is reduced significantly, whereas the hydrodynamic particle diameter is hardly affected.

Figure 2 demonstrates clearly that with TMB magnetite nanoparticle diameters can be determined in dense, highly light scattering media with high sensitivity $\left(170 \mathrm{uM} \mathrm{Fe}_{3} \mathrm{O}_{4}\right)$ and low dispersion of the data. In particular, we want to stress that, while the color in the photo clearly indicates strong fluctuations in particle concentration along the gel lane, the electrophoretic mobility vs. hydrodynamic diameter does not deviate from a linear behavior. This suggests that fluctuations in the number of light scattering events do not affect the determination of the particle diameter with TMB, even though we are in the multiple scattering regime. In fact, cross-check experiments have shown that the light depolarization caused by the gel and fluctuations in the particle concentration do not affect the hydrodynamic diameter measurements significantly, and hydrodynamic diameter fluctuations lie within only $1.7 \%$ (figure S4 in the Supplementary Data).

We can see in figure 2 that obtained diameter values are relatively high. Theoretical studies suggested that the main contribution to the birefringence signal comes from the orientation of pre-existing dimers and anisometric aggregates [25, 26], since both shape anisotropy and intrinsic optical anisotropy of nominally spherical magnetite nanoparticle monomers are low. The fact that the transfer of nanoparticles from organic solvents to water based solutions usually involves the formation of some small aggregates supports this suggestion [27].

We performed microscopy measurements to check for the presence of aggregates. Transmission Electron Microscopy (TEM) images of PMAO functionalized nanoparticles show mainly 
monodisperse nanoparticles, but suggest the presence of some aggregates (figure 3a). However, organic material is not visible in these images, and it is not clear whether several particles are enwrapped in a common polymer shell. With Amplitude Modulation Atomic Force Microscopy (AM-AFM) organic material is imaged, and different materials can be differentiated through the excitation frequency shift. Aggregates appearing as one single cluster in the topography image (figure $3 \mathrm{~b}$, left) can be assigned the number of particles constituting them from the excitation frequency shift image (figure $3 \mathrm{~b}$, right). AFM inspection revealed that, although a large part of the particles is monodispersed, a considerable fraction of the particles is present in the form of dimers, trimers and higher order aggregates which mainly contribute to the birefringence.

a
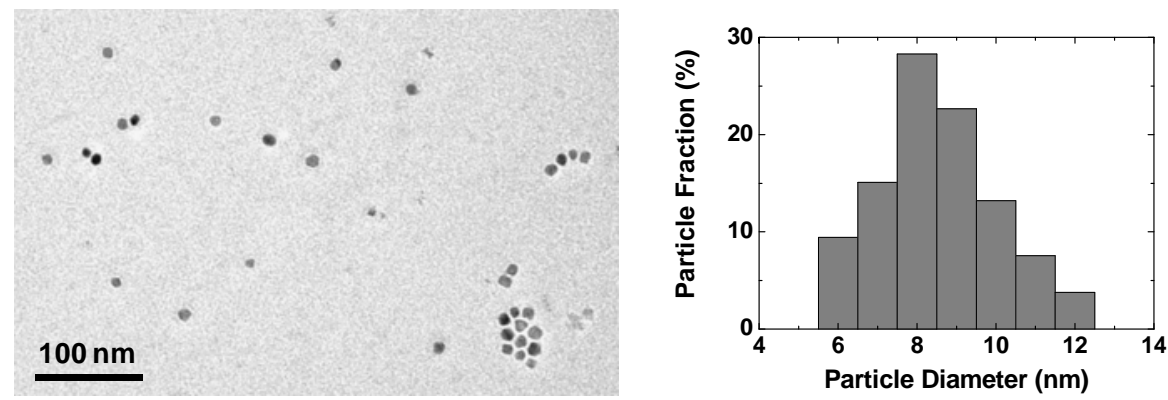

b
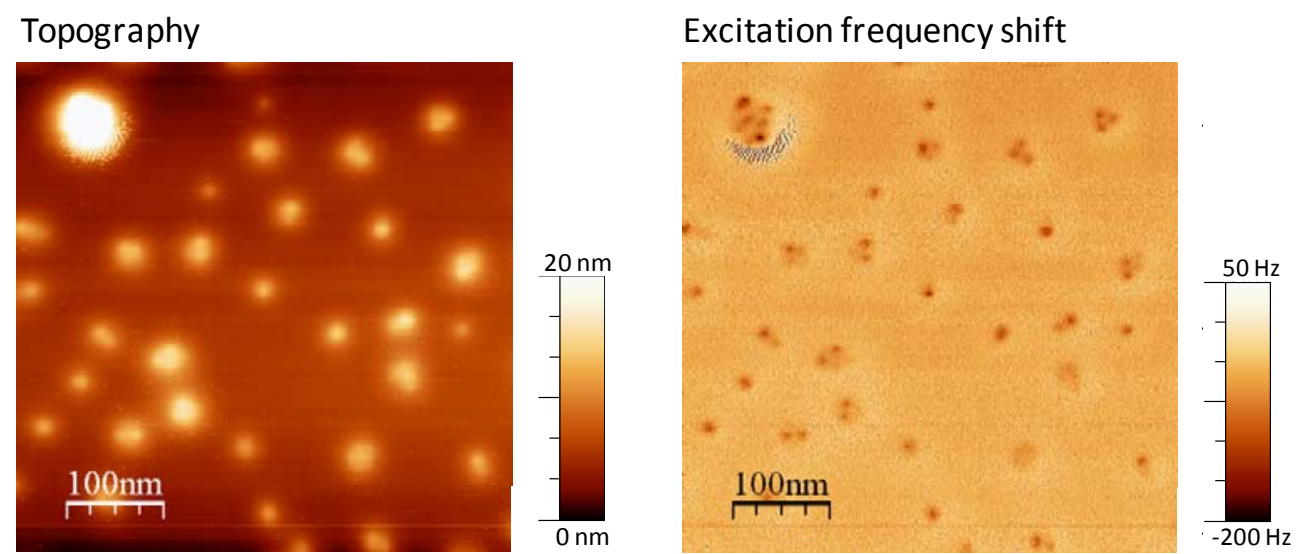

Figure 3. Characterization of nanoparticle size, shape and aggregation. (a) Typical TEM image showing both size and shape distribution of the nanoparticles' inorganic $\mathrm{Fe}_{3} \mathrm{O}_{4}$ core. Images with low nanoparticle density as shown here suggest the presence of some aggregates. The particle size distribution was determined from about 100 particles (figure S1 shows a TEM 
image with high particle density); (b) AFM images confirm that, indeed, particle dimers, trimers and higher order aggregates are present.

In order to cross-check the measured hydrodynamic diameter values with an established technique, we performed ex situ Dynamic Light Scattering (DLS) measurements. DLS is a widely used method for measuring the hydrodynamic diameter distribution of particles and their aggregates due to its versatility, based on the fact that it detects any kind of particles (of sizes between approximately $1 \mathrm{~nm}$ and $10 \mathrm{um}$ ) present in very dilute suspensions, independently of their physical (optical, magnetic...) properties. The insensitiveness to a certain particle property, however, makes the technique unsuitable for determining the sizes of particles suspended in serum or even embedded in dense, highly light scattering media like cell tissue, and particle diameters can only be characterized ex situ. Therefore, measurements were performed on the PMAO functionalized particles after they had diffused out of the $2 \%$ gel into buffer solution.

DLS shows (figure 4) that two populations remain throughout the entire gel lane, but each population shifts to lower diameter values with increasing traversed distance. In table 1 the mean hydrodynamic diameters of the two populations are listed. We attribute the two DLS populations to particle monomers $\left(d_{\mathrm{hyd}, 1}\right)$ and aggregates $\left(d_{\mathrm{hyd}, 2}\right)$. The presence of these two populations throughout the whole gel lane demonstrates that agarose gel electrophoresis does not yield a complete separation of nanoparticle monomers from dimers, possibly due to the dimers moving with one particle in the slip stream of the other. It should be noted that larger particles yield a higher contribution to the scattered light intensity, since the scattered light intensity depends on the particle diameter to the sixth power. Thus, although in number only few large particles are present, the scattered light intensity of these large particles is relatively high. In fact, a conversion of the size distribution by intensity (shown in figure 4) to the size distribution by number (shown in figure S5 of the Supplementary Data) demonstrates that the number of aggregates is actually very low. This confirms the sensitivity of light scattering 
techniques to particle aggregation.

a

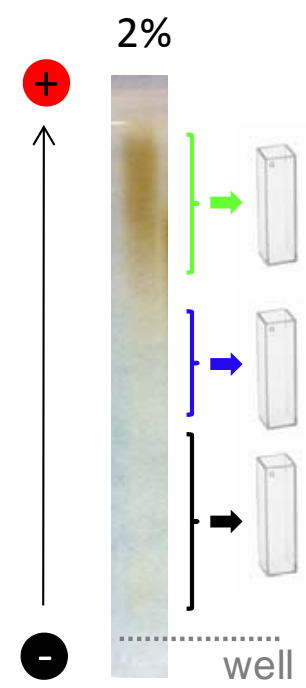

b

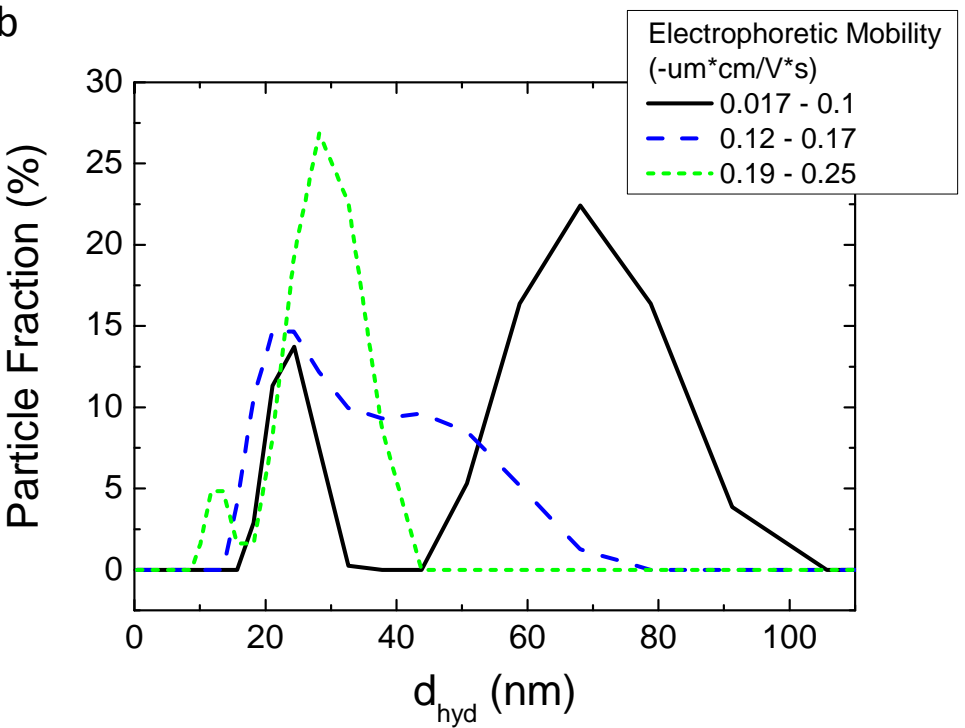

Figure 4. Hydrodynamic nanoparticle diameters determined ex situ by Dynamic Light Scattering (DLS). (a) Photograph of the $2 \% \mathrm{w} / \mathrm{v}$ agarose gel after applying $5 \mathrm{~V} / \mathrm{cm}$ during 110 min (NPs functionalized with PMAO). The ranges of the gel from which particles were collected for the DLS measurements are indicated. (b) Hydrodynamic diameter distribution (by intensity) of the particles after separation through gel electrophoresis, obtained by DLS after particle diffusion out of the gel into buffer solution.

TABLE 1. Mean hydrodynamic diameters of the two populations detected by DLS after nanoparticles functionalized with PMAO had diffused out of the $2 \%$ agarose gel into buffer solution.

\begin{tabular}{lll}
\hline $\begin{array}{l}\text { Mobility } \\
\left(-\mu \mathrm{m} \mathrm{cm} \mathrm{V}^{-1} \mathrm{~s}^{-1}\right)\end{array}$ & $\begin{array}{l}d_{\text {hyd,1 }} \\
(\mathrm{nm})\end{array}$ & $\begin{array}{l}d_{\text {hyd,2 }} \\
(\mathrm{nm})\end{array}$ \\
\hline $0.017-0.1$ & 22 & 70 \\
$0.12-0.17$ & 22 & 45 \\
$0.19-0.25$ & 13 & 30 \\
\hline
\end{tabular}


The comparison of hydrodynamic diameters determined by TMB and DLS gives the definite evidence that TMB does not detect nanoparticle monomers: Monomer nanoparticles have DLS hydrodynamic diameters $<40 \mathrm{~nm}$, whereas the lowest hydrodynamic particle diameter detected with TMB is $55 \mathrm{~nm}$. This demonstrates that dimers and higher aggregates of spherical magnetite nanoparticles yield the main contribution to the birefringence signal, while monomers do not contribute significantly - in agreement with theoretical results which proposed the orientation of pre-existing aggregates to give the main contribution to the birefringence signal $[25,26]$. The particle size distribution in equation (2) is then actually the distribution of the aggregate size and a log-normal distribution is justified. The inter-particle distance is approximately 1.5 times the particle diameter, since the polymer is covering each particle (see TEM and AFM micrographs in figure 3). Due to this low inter-particle distance dipolar interactions are important and capable of giving rise to an effective torque to the aggregate in the external pulsed magnetic field. On the other hand, magnetic field induced aggregation of our superparamagnetic nanoparticles has not been observed, as expected.

For biosensor applications, specific and controlled aggregation of adequately functionalized magnetic particles allows for detecting biomolecules with high sensitivity when detection schemes are used which are intrinsically selective to particle clusters with respect to single particles [28]. Such a detection scheme could make use of the optical anisotropy which is only induced in a suspension of dimers or aggregates of spherical magnetic nanoparticles upon the application of a magnetic field, while suspensions of individual spherical nanoparticles remain optically isotropic. We propose that, in principle, birefringence constitutes a sensitive means for detecting molecular recognition through specific and controlled aggregation of spherical magnetite nanoparticles in dense, highly light scattering media. In case unspecific aggregation cannot be completely excluded, molecular recognition can be monitored through the hydrodynamic particle diameter increase, which has been demonstrated before [8-10]. Then, however, only the fraction of anisometric aggregated NPs contributes to the birefringence, and 
sensitivity depends on this parameter. By using elongated magnetic nanoparticles sensitivity should increase significantly [26].

By comparing TMB and DLS measurements we can also deduce that TMB does not detect aggregates of small nanoparticles: Particles that were extracted far from the well (with high mobility) present a second peak (due to aggregates) in DLS with diameters $<45 \mathrm{~nm}$, which were not detected with TMB. This might be due to the weak magnetic dipolar interaction of particles below $5 \mathrm{~nm}$ (the magnetic moment of the iron oxide NPs decreases significantly when the diameter falls below $5 \mathrm{~nm}$ [29]) which impedes the alignment of these aggregates with the magnetic field. In consequence, in the case of spherical NPs, the birefringence amplitude does not depend on the total NP concentration, measured through the optical absorption, but only on the dimers' and anisometric aggregates' contribution of not too small nanoparticles (see also figure S6 in the Supplementary Data).

Generally, differences in the hydrodynamic diameters determined by DLS and TMB are expected since different techniques are sensitive to different particle properties and none of the results are inherently correct or absolute. While for TMB the rotational component of the Brownian motion is decisive, in DLS it is the translational component. Depending on the shape of the objects this may lead to significant variations in the determined hydrodynamic diameter. In this case Depolarized Dynamic Light Scattering would be more appropriate for comparison studies. Nonetheless, studies comparing effective particle sizes obtained with different techniques have shown that variations are especially pronounced when particles are functionalized with long and complex surfactant molecules or polymer layers [30] as it is the case here. Then the approximation that the hydrodynamic diameter is the inorganic core diameter plus two times the thickness of the organic layer breaks down and both the steric conformation of the organic molecules and the hydration influence the hydrodynamic diameter significantly. Nevertheless, always when using one method consistently, relative changes in hydrodynamic diameters are meaningful. 
Since TMB only detects not too small magnetic NPs with shape anisotropy, it is not an appropriate method for the hydrodynamic particle size determination in general as is DLS. The strength of the technique lies rather in taking advantage of distinguishing particle features like their magnetic properties and shape anisotropy which allow for monitoring the particles' hydrodynamic diameter and detecting molecular recognition in highly dispersive media in situ, for example in biological tissue where proteins or other present molecules may adsorb and particles may aggregate, thus altering the performance of the particles through changes in their functionality and size. Standard Dynamic Light Scattering, however, can only be used in very dilute nanoparticle suspensions and not for nanoparticles suspended in serum or even embedded in complex scattering media like cell tissue. For these applications TMB presents an inexpensive and easy to build solution.

\section{Conclusions}

Transient Magnetic Birefringence (TMB) is a sensitive tool for monitoring the hydrodynamic diameters of anisometric magnetic nanoparticles in dense media with strong background light scattering. The technique was applied to the in situ measurement of hydrodynamic diameters of spherical $\mathrm{Fe}_{3} \mathrm{O}_{4}$ nanoparticles after their electrophoretic separation in agarose gels. Although multiple light scattering in dense media diminishes the polarization of the transmitted light, in this work we show that with TMB reliable results are obtained even for dense and highly scattering media such as an agarose gel. This presents a proof of concept in a model system that scatters light in a similar way as a more complex biological medium but where particle-matrix interactions are low. In fact, we did not observe a significant effect of gel-particle interactions on the rotational particle diffusion. In our systematic study, comparing the hydrodynamic diameter values obtained in situ by TMB with those obtained ex situ by Dynamic Light Scattering (DLS), and correlating both to observations made in microscopy studies (TEM and AFM), we demonstrate that the main contribution to the birefringence signal comes from dimers 
and small optically anisotropic aggregates of spherical $\mathrm{Fe}_{3} \mathrm{O}_{4}$ nanoparticles. Indeed, monomers do not yield any appreciable birefringence signal. Therefore, magnetically induced birefringence can be used in sensitive molecular recognition applications, where specific and controlled dimerization of functionalized magnetite particles can be detected through a significant rise in birefringence. These results pave the way to use magnetically induced birefringence for studying possible interactions of the nanoparticles with biological media like living cells and tissue.

Acknowledgment. This work has been supported through the projects NAN2004-09125-C0702, PROYECTO INTRAMURAL DE FRONTERA DE CSIC Ref. 200550F0172, PROFIT BIOSENSE FIT-010000-2006-98, CTQ2008-03739/PPQ and the Starting Grant-ERC NANOPUZZLE. M.K. is grateful to the Spanish Council for Scientific Research for an I3P fellowship. M.M. acknowledges support through the CONSOLIDER-NANOBIOMED project. We also thank P. Morales for fruitful discussions and her help with the DLS measurements and I. Echaniz for technical support. 


\section{References}

[1] Pankhurst Q A, Thanh N K T, Jones S K and Dobson J 2009 J. Phys. DAppl. Phys. 42224001

[2] Gupta A K and Gupta M 2005 Biomaterials 26 3995-4021

[3] Rocker C, Potzl M, Zhang F, Parak W J and Nienhaus G U 2009 Nature Nanotechnol. 4 577-80

[4] Verma A and Stellacci F 2010 Small 6 12-21

[5] Pons T, Uyeda H T, Medintz I L and Mattoussi H 2006 J. Phys. Chem. B, 110 20308-16

[6] Parak W J, Pellegrino T, Micheel C M, Gerion D, Williams S C and Alivisatos A P 2003 Nano Lett. 3 33-36

[7] Israelachvili J and Wennerstrom H 1996 Nature 379 219-25

[8] Wilhelm C, Gazeau F, Roger J, Pons J N, Salis M F, Perzynski R and Bacri J C 2002 Phys. Rev. E 65031404

[9] Romanus E, Gross C, Glockl G, Weber P and Weitschies W 2002 J. Magn. Magn. Mater. 252 384-86

[10] Aurich K, Nagel S, Heister E and Weitschies W 2008 Nanotechnology 19 505102

[11] Davies H W and Llewellyn J P 1979 J. Phys. D-Appl. Phys. 12 1357-63

[12] Bacri J C, Perzynski R, Salin D and Servais J 1987 Journal De Physique 48 1385-91

[13] Benoit H 1951 Ann. de Phys. 6 561-609

[14] Badoz J 1956 Journal De Physique Et Le Radium 17 A143-49

[15] Kiss L B, Soderlund J, Niklasson G A and Granqvist C G 1999 Nanotechnology 10 25-28

[16] Limpert E, Stahel W A and Abbt M 2001 Bioscience 51 341-52

[17] Sun S H, Zeng H, Robinson D B, Raoux S, Rice P M, Wang S X and Li G $X 2004$ Journal of the American Chemical Society 126 273-79

[18] Pellegrino T, Manna L, Kudera S, Liedl T, Koktysh D, Rogach A L, Keller S, Radler J, Natile G and Parak W J 2004 Nano Lett. 4 703-07

[19] Moros M, Pelaz B, Lopez-Larrubia P, Garcia-Martin M L, Grazu V and de la Fuente J M 2010 Nanoscale 2 1746-55

[20] Yu W W, Chang E, Sayes C M, Drezek R and Colvin V L 2006 Nanotechnology 17 4483-87

[21] Pyell U 2010 Electrophoresis 31 814-31

[22] Hanauer M, Pierrat S, Zins I, Lotz A and Sonnichsen C 2007 Nano Lett. 7 2881-85

[23] Zanchet D, Micheel C M, Parak W J, Gerion D, Williams S C and Alivisatos A P 2002 J. Phys. Chem. B 106 11758-63

[24] Sperling R A, Pellegrino T, Li J K, Chang W H and Parak W J 2006 Adv. Funct. Mater. 16 943-48

[25] Scholten P C 1980 IEEE Trans. Magn. 16 221-25

[26] Xu M and Ridler P J 1997 J. Appl. Phys. 82 326-32

[27] Bagaria H G, Kini G C and Wong M S 2010 J. Phys. Chem. C 114 19901-07

[28] Ranzoni A, Schleipen J J H B, van ljzendoorn L J and Prins M W J 2011 Nano Lett. 11 2017-22 
[29] Rebolledo A F, Fuertes A B, Gonzalez-Carreno T, Sevilla M, ValdesSolis T and Tartaj P. 2008 Small 4 254-61

[30] Sperling R A, Liedl T, Duhr S, Kudera S, Zanella M, Lin C A J, Chang W H, Braun D and Parak W J 2007 J. Phys. Chem. C 111 11552-59 